\title{
Multilinguales
}

\section{L'écriture réflexive dans LA KAHENA de Salim Bachi}

Reflexive writing in LA KAHENA of Salim Bachi

$$
\text { لانعكابة إسية في "كاهنة" لسليم باشي }
$$

\section{Leila Oulebsir et Sabrina Fatmi}

\section{OpenEdition}

Journals

Édition électronique

URL : http://journals.openedition.org/multilinguales/1049

DOI : $10.4000 /$ multilinguales.1049

ISSN : 2335-1853

\section{Éditeur}

Université Abderrahmane Mira - Bejaia

\section{Référence électronique}

Leila Oulebsir et Sabrina Fatmi, "L'écriture réflexive dans LA KAHENA de Salim Bachi », Multilinguales [En ligne], 9 | 2018, mis en ligne le 01 juin 2018, consulté le 17 août 2020. URL : http:// journals.openedition.org/multilinguales/1049; DOI : https://doi.org/10.4000/multilinguales.1049

Ce document a été généré automatiquement le 17 août 2020.

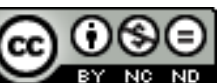

Multilinguales est mise à disposition selon les termes de la Licence Creative Commons Attribution Pas d'Utilisation Commerciale - Pas de Modification 4.0 International 


\title{
L'écriture réflexive dans LA KAHENA de Salim Bachi
}

Reflexive writing in LA KAHENA of Salim Bachi

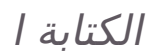 \\ لانعكاسية في "كاهنة" لسليم باشي
}

Leila Oulebsir et Sabrina Fatmi

1 La littérature dite moderne défend, dans une conception structuraliste, son orientation créatrice dans un processus réflexif incessant, en elle-même et pour elle-même. En réfléchissant son agencement et son développement elle s'écarte ouvertement de toute vraisemblance, en dehors des représentations référentielles et des codes mimétiques. Cette orientation littéraire, qui traduit une prise de conscience évidente de la déficience du langage à cerner une réalité insaisissable, fait de la matière verbale une création artistique et une jouissance où les mots, au sens de Barthes, «ne sont plus conçus illusoirement comme de simples instruments, ils sont lancés comme des projections, des explosions, des vibrations, des machineries, des saveurs» (1978: 20). En effet, la notion de réflexivité, et particulièrement celle de la mise en abyme ${ }^{2}$, connaît une renommée considérable dans tous les domaines artistiques, notamment dans les années soixante. C'est l'écrivain et critique André Gide qui, le premier, introduit dans son journal (1948) la désignation définitive de cette notion, dans l'usage littéraire, en proposant l'image $\mathrm{du}$ «blason qui consiste, dans le premier, à mettre un second en abyme» (cité par Dällenbach, $1977: 15)$.

2 Néanmoins, face à la perplexité conceptuelle qui désigne ce miroir intérieur du récit, Dällenbach propose un examen systématique de ce procédé dans son ouvrage Le récit spéculaire, Essai sur la mise en abyme (1977), qui s'effectue selon une dimension triptyque (heuristique, théorique et historique). Il parvient, de la sorte à hisser cette notion à son statut de théorie littéraire qu'il définit comme « tout miroir interne réfléchissant l'ensemble du récit par réduplication simple, répétée ou spécieuse » (1977:52).

3 Cette tripartite donne lieu, d'une part, à une réflexion fictionnelle (de l'énoncé ou du contenu), qui se présente sous forme d'analogie ou de parallélisme entre le récit 
enchâssé et le récit enchâssant. D'autre part, elle reproduit une réflexion de l'énonciation qui reflète les instances narratives, et donc l'acte ainsi que le contexte de production et de communication. Cependant, elle dessine également une troisième partie, et c'est le but de notre analyse dans ce présent article, qui est la réflexion du code dans le récit. Cette forme de mise en abyme suppose donc une sorte de miroir qui renvoie à la structure, à l'agencement formel du texte et à son "aspect littéral d'organisation signifiante» (Dällenbach, $1977: 123)$. En ce sens, l'idée première de ce critique littéraire est de peindre cette notion spéculaire comme un processus de surplus sémantique, qui permet au récit de se contempler lui-même en tant que son propre objet d'étude :

(...) l'énoncé supportant la réflexivité fonctionne [alors] au moins sur deux niveaux : celui du récit, où il continue de signifier comme tout autre énoncé, et celui de la réflexion où il intervient comme élément d'une métasignification permettant au récit de se prendre pour thème (Dällenbach, $1977: 62$ ).

4 Notre étude se basera donc, essentiellement, sur la typologie étudiée dans cet ouvrage fondateur de Dällenbach et s'attache, particulièrement, à l'examen des divers jeux de miroir aux niveaux synchronique et diachronique agencés dans l'économie du roman de Salim Bachi ${ }^{3}$, LA KAHENA (2003). Cependant, nous ferons également appel à l'ouvrage Mythologies (1957) de Roland Barthes afin de pouvoir aborder la profusion de la substance mythique dans le texte, l'une des cosmogonies du récit bachilien ${ }^{4}$, dans son rapport étroit à l'imaginaire collectif. Nous l'examinerons comme une médiatisation intelligible du langage, entre le texte et son dehors, et comme un questionnement sensible sur l'être humain, la femme en particulier, sa condition et sa destinée difficilement abordable par le biais d'un raisonnement rationnel. Cette perspective nous permettra de développer, d'une part, les pratiques réflexives auxquelles s'apprête le code ${ }^{5} \mathrm{du}$ récit et de réfléchir, d'autre part, sur une probable mise en place d'un pont mythique ${ }^{6}$, fécond et énergique, entre l'univers de la fiction et celui du réel. L'examen de cette filiation conjointe va contribuer, par ailleurs, à distinguer le mouvement et la présence signifiante de la figure féminine de La Kahéna ${ }^{7}$, symbole d'une Algérie rebelle et insoumise, en tant que valeur et empreinte sociale dans le récit. Nos interrogations seront principalement axées sur les stratégies, les fonctions et les enjeux du phénomène réflexif de la mise en abyme et, corrélativement, sur la cohabitation subtile d'une dimension mythique interceptée comme une ultra-signification, en reprenant Barthes, derrière laquelle se détermine «à la fois sens et forme » (1957:201).

5 Pour guider notre réflexion, nous prévoyons deux hypothèses fondamentales qui stipulent que : l'impact de la mise en abyme collabore, en premier lieu, à l'élaboration du tissu textuel, sémantique et esthétique dans l'œuvre ; elle affiche, de ce fait, un effet narcissique excessif qui inscrit une dimension parodique palpable dans le texte. En second lieu, le récit mythique accomplit sous forme de métalangage ${ }^{8}$, à travers la projection de la figure emblématique de La Kahéna, la représentation conjointe du destin de la femme rebelle qui est étroitement rattachée à l'âme et l'esprit d'un pays martyrisé. Nous tenterons, en somme, de rendre compte de la singularité scripturale propre à l'auteur qui pourrait, éventuellement, permettre au langage de saisir une vision du monde et de la société, au-delà d'un jeu égocentrique outrancier du texte. L'écriture ébranlerait, de ce fait, l'aspect redondant et tautologique ${ }^{9}$ du texte sans se défaire, pour autant, de son caractère fictionnel.

6 Succinctement, ce roman déplie un récit qui foisonne dans l'imagination $d u$ personnage central, Hamid Kaïm, et se déroule dans l'énigmatique résidence de 
Bergagna nommée La Kahéna. Symbole de la révolte et de la résistance, ce nom lui a été assigné à l'instar de la grande reine berbère; un mythe historique qui s'élève dans l'imaginaire de son peuple "contre l'oubli" $(142)^{10}$ et contre les envahisseurs, usurpateurs de la mémoire et de l'identité. Le récit entraine le narrateur en délire, qui agonise dans une chambre de la villa du colon, dans un tissu narratif complexe de trois nuits, sur le modèle des Mille et une Nuits. Ce dernier, symbolise une Schéhérazade de pacotille qui prend à son tour les rênes du récit encadrant et enchâsse, par la même, les multiples histoires de son interlocuteur où se croisent, au fil des siècles, plusieurs générations au destin tragique.

\section{La réflexion métatextuelle}

7 La réflexion métatextuelle est assumée par des métarécits ${ }^{11}$ qui concrétisent le retour de l'œuvre sur soi et qui manifestent une valeur instructive et programmatique "en rendant intelligible le mode de fonctionnement du récit (...) sans pour autant mimer le texte qui s'y conforme » (Dällenbach, 1977 : 127). Elle assume alors le rôle d'édificateur de l'œuvre en attirant l'attention du lecteur vers son processus de création, dans une allégorisation parfaite du roman et de l'écriture. Ainsi, la métaphore du code textuel, qui s'impose d'emblée dans notre corpus est représentée, d'un point de vue spatial, par la villa de Bergagna, éponyme du roman de Bachi LA KAHENA. Une appellation reprise par l'auteur à la reine berbère des Aurès qui, en narrativisant de manière plus ou moins précise l'activité de son écriture, désigne une " étrange dénomination pour une maison de colon » (20). La réflexion métatextuelle est donc savamment assurée, d'entrée de jeu, par la somptueuse maison qui, à l'image d'une déesse protectrice suscitant le respect et la crainte, récupère aussitôt le titre du roman en le renfermant: "La Kahéna et la symbolique qui s'y rattachait (...) était l'épicentre d'où partaient les vagues concentriques de la révolte» (263).

En réfléchissant ainsi le texte sous une forme architecturale, cette demeure matérialise un fragment textuel censé inclure l'œuvre et la révéler afin de désigner ses dispositifs d'engendrement. Elle reflète, dès lors, l'agencement du récit en proposant au lecteur un plan d'orientation loin des pratiques lectoriales confortables et passives: «(...) un lieu hors du monde où le temps s'abolirait en une éternité de délice " (72). Partant, cette mise en abyme dite aporistique ou spécieuse s'impose, à première vue, comme un miroir judicieux qui contient l'œuvre et qui l'enchâsse en caricaturant le processus de création de «l'œuvre même [par] (identité)» (Dällenbach, 1977 : 142). Ce miroitement textuel semble ainsi posséder un puissant pouvoir de gestion sur l'économie du récit en réfléchissant «le code des codes » (ibid. : 138), démêlant ainsi, au fil de la lecture, son mystère et celui $\mathrm{du}$ texte. Cependant, ce mouvement réflexif du texte dénonce, en parallèle, le foisonnement de ces jeux rotatifs en les exhibant à l'excès bravant, de la sorte, une "Transformation ludique d'un texte singulier» (Genette, 1982: 202). Ces jeux réalisent, visiblement, la mise en valeur d'une apparence parodique ${ }^{12}$, révoquant ainsi l'aspect récursif du texte, qui n'a pour ultime projet que de leurrer le lecteur désorienté dans sa quête infinie du sens : "Quête inaboutie, forme vicieusement circulaire, rhétorique dérisoire, écriture à la dérive " (Dällenbach, $1977:$ 137). L'auteur semble ainsi entretenir un discours carnavalesque caricaturant consciemment, et de manière abusive, une écriture dénuée de son sens. Il tente ainsi, forcément, une reconsidération de l'aspect narcissique du texte en suggérant une volonté de ce dernier de déboucher sur autre 
chose que lui-même. Dällenbach n'a pourtant pas manqué d'aborder la question sur cette pratique démesurée de la mise en abyme qu'il considère comme un «saut dans la Révolution et la Jouissance » (1977 : 210). Il examinait d'ores et déjà, globalement dans son ouvrage, les enjeux d'une utilisation exagérée du procédé et l'avenir que réserve le refus catégorique de tout mimétisme:»conforme aux sanctions de l'Histoire (...) toute tendance excessive (ici une littérature du Signifiant) appelle la tendance inverse (une littérature du Signifié, de la Référence de la Pulsion » (1977 : 210).

Tout compte fait, cette configuration du miroir réflexif, où l'on retrouve généralement des titres et des personnages quasiment identiques, installe une relation identitaire étroite entre l'histoire du micro et du macro-récit, telle une allégorie exemplaire du mouvement textuel. Toutefois, nous pourrions soutenir aisément, à ce stade, une volonté de démystification apparente du retour du texte sur soi, savamment représentée par la forme architecturale de la résidence du colon, qui s'acharne à mimer une circularité immodérée "Comme si le cercle tendait lui-même à s'ouvrir sur un futur, en fonction des forces en cuvres qu'il abrite (...) pour rejoindre des forces de l'avenir, des forces cosmiques (...) rejoindre le Monde» (Deleuze et Guattari, $1980: 383$ ). Signalons que Marc Gontard fait référence à cette particularité qui relève de l'écriture postmoderne ${ }^{13}$ où "le dispositif métatextuel révèle un mode de réflexivité distinct de la mise en abyme instrumentalisée par le Nouveau Roman, [et] où le texte tout en s'écrivant se commente dans le métatexte» (2013: 88).

\section{La mise en abyme transcendantale}

10 Cette forme de mise en abyme désigne une réflexion transcendantale quand elle dévoile dans le récit «ce qui tout à la fois l'origine, le finalise, le fonde, l'unifie et en fixe les conditions a priori de possibilité» (Dällenbach, 1977 : 131). Elle divulgue donc, à la fois, l'aspect formel du texte ainsi que son aspect fictionnel; elle évolue nécessairement d'une réflexion métatextuelle à une mise en abyme transcendantale qui " s'accompagne toujours (...) de la réflexion subsidiaire de l'énoncé et du principe de fonctionnement du récit » (ibid. : 139).

\section{La réflexion transcendantale via le journal de $\mathrm{H}$. Kaïm}

11 Concernant cette forme de réflexion, l'exemple qui s'impose concrètement est celui des manuscrits trouvés, cachés dans la maison de Bergagna, dont le journal du père de H. Kaïm. Ces derniers sont soigneusement dissimulés dans un oiseau empaillé que le colon maltais a rapporté de son voyage en Amazonie. Le fameux journal contient quasiment toute la trame du récit et se présente, sous forme de miniature, comme un micro-récit enchâssé dans un récit cadre; il évolue pourtant autour de ce noyau fondamental et envahit ensuite tout le roman:» maintenant le manuscrit qu'il tenait [le narrateur] entre ses mains prenait un poids considérable; ses quelques paroles, émises voilà plus de vingt ans, acquéraient un volume impressionnant» (167). Cet exemple matérialise aisément un schéma rotatif du récit regagnant fatalement, dans une circularité vicieuse, son point de départ à savoir l'hôpital où agonise le personnage principal, Hamid Kaïm. Le récit du journal s'affirme ainsi, pleinement, comme le résultat de l'imagination du protagoniste en délire, sur son lit de mort, qui ne cesse de multiplier les histoires dans des hallucinations excentriques. 
Ce foisonnement d'histoires est cependant retransmis dans un emboitement incessant, à l'image des Mille et une Nuits, par son auditrice/narratrice et amante, nommée Schéhérazade, qui n'attend que la fin du récit pour lui ôter la vie, semblable à «la mort qui ceuvre dans le silence, la mort qui gratte du papier, enfile les mots comme un collier de bimbeloterie » (280). Le journal se manifeste ainsi comme le miroir du livre en train de se construire et de prendre forme "amplifiant le drame ou la tragédie, selon le point de vue adopté» (128). Or, par un glissement progressif de la métaphore, il devient le corps de l'écriture et des êtres qui s'y meuvent; une structure narrative indéfectible où l'histoire de la belliqueuse reine berbère, relatée en bribes par le narrateur, brouille toutes les frontières du récit narcissique : «porté par sa propension à enrober la réalité d'un voile propice au séjour de la parole. Le mythe prenait [ainsi] naissance dans sa bouche» (53). Associé à l'histoire de la famille de Bergagna et du père de Kaïm, le mythe de La Kahéna s'impose et s'érige donc comme une force gravitationnelle, qui dirige le récit, afin de transmettre l'esprit douloureux d'une réalité tragique. L'auteur exploite et prolonge ainsi un sujet souvent sollicité par d'autres auteurs, tant algériens que maghrébins, en conciliant réalité et fiction, conte et mythes, Histoire et histoire : «(...) cette reine berbère survivait dans les mémoires en raison de son acharnement à vaincre l'envahisseur » (20). La présence mythique dans le texte s'affirme ainsi telle une parole qui, selon Barthes,

n'a pas la vérité pour sanction : rien ne l'empêche d'être un alibi perpétuel : il lui suffit que son signifiant ait deux faces pour disposer toujours d'un ailleurs : le sens est toujours là pour présenter la forme; la forme est toujours là pour distancer le sens. Et il n'y a jamais contradiction (1957: 196).

13 Tout bien considéré, ce manuscrit retrouvé, qui déroule une histoire indéterminée et inachevée, dévoile à la fois l'organisation formelle du texte ainsi que son aspect fictionnel. Il matérialise, de la sorte, une mise en abyme métatextuelle qui se transpose, forcément, en une réflexion transcendantale. Le journal tenu par le père de Kaïm témoigne ouvertement du caractère allégorique du livre, qui se crée en même temps qu'il s'écrit. Il dessine, de ce fait, le prototype d'une œuvre analogue, par similitude, puisque la réduplication est ici de type simple, sachant que cette similitude des récits enchâssés et enchâssant est inévitablement vague et approximative.

\section{La réflexion transcendantale via la villa}

14 Comme nous l'avons signalé au préalable, le traitement du personnage de La Kahéna est particulier, dans le sens où il est substitué à la résidence du colon maltais. Cette habitation, qui se conçoit comme le reflet patent du texte et de son activité, était donc "vivante: un visage, la façade; une crinière touffue» (64). Mais, nous verrons également qu'elle peut constituer un réceptacle de l'histoire fictionnelle au niveau de l'énoncé, puisque la personnification symbolique de cette demeure dessine, manifestement, une réflexion transcendantale. Ses multiples descriptions s'appliquent, en effet, à refléter un miroir inversé des événements réels, interceptés et fictionnalisés : «Le symbolisme externe du roman tend [donc] à se réfléchir dans un symbolisme interne, certaines parties jouant, par rapport à l'ensemble, le même rôle que celui-ci par rapport à la réalité» (Butor, 1969 : 12). Effectivement, en évoquant un mythe berbéro-Maghrébin, la villa réincarne la figure féminine ancestrale d'une reine résistante qui "persistait dans la mémoire des indigènes comme la femme qui se refusa à eux et qui se dressa à rebours de la conquête musulmane " (110). Personnage historique et souvent problématique, l'Histoire garde d'elle l'image d'une reine qui a su défendre son pays, créer un état, ériger une politique 
et commander des armées. La mémoire de La Kahéna, son âme et sa grandeur se matérialise, de la sorte, à travers une habitation somptueuse où l'auteur fait valoir, vraisemblablement, une volonté concrète de féminiser son roman comme "une, redoutable, destinée à symboliser son règne et sa puissance " (54). Néanmoins, en portant le nom d'une femme mémorable et hérö̈que, cette villa convoque et réfléchit, par transcendance, une longue Histoire tragique et amnésique profondément ancrée dans les mémoires collectives :

les armées se déversaient de La Mecque sur le monde à peine nubile, encore engorgé de son antiquité, adolescent, se remodelant au galop, subissant sa première mue, sa première défaite, mais la terre était plus vieille, et ces armées du Croissant la parcouraient dans l'ignorance du passé, des conquêtes et des peuples: elles furent surprises par l'acharnement de la reine juive à défendre son royaume (...) redoutant pendant les siècles à venir le réveil de la première femme qui leur résista. (20).

La maison se métamorphose, par conséquent, au bénéfice de sa mythification à l'image d'une reine-guerrière dont le nom a su traverser les âges. Le récit va donc œuvrer dans une double action qui figure une femme/villa et qui apparait comme un support phénoménal et fécond de l'imaginaire du narrateur. En effet, de par son combat politicohéroïque, la reine berbère est ainsi élevée au rang de mythe sacré ${ }^{14}$, à valeur sémiotique incontestable, qui permet au texte de saisir des sens hétérogènes et pluriels. Ce procédé pourrait donc participer, entre autres, à l'inscription du texte dans un processus de déconstruction du phallocentrisme traditionnel, qui impose la domination despotique d'un être par son semblable et, par conséquent, de la femme par l'homme. C'est pourquoi la fascination que procure l'image mythique de La Kahéna divulgue une exaltation et une valeur fondamentales qui exécutent, dans le récit, le reflet d'une consécration de la gent féminine; élevée au rang de reine, elle "instaurait [ainsi] ses propres règles 》 (18).

Réactualisé par l'auteur, ce mythe à la sève intacte est donc adopté à des fins romanesques et allégoriques " réduisant [ainsi] l'écart entre le monde et [l] es songes » (297); il demeure un aspect du langage garant de la résistance, de l'identité et de la mémoire "que le plus fou des poètes n'eut jamais osé inventer » (279). Cependant, c'est au lecteur qu'échoit la responsabilité de "rattacher le schème mythique à une histoire générale, [d'] expliquer comment il répond à l'intérêt d'une société définie (...) [et] qui doit en révéler la fonction essentielle» (Barthes, $1957: 202)$. Ainsi, c'est à l'image du langage que l'espace même du mythe s'engendre à travers la fiction et, par là même, c'est la structure textuelle qui se crée et prend effet à travers lui : «il "donne à penser" et irréalise au profit d'un signifié inépuisable la teneur du récit» (Dällenbach, 1977:82). Du récit au mythe comme récit, s'installe alors un processus d'amplification et d'enrichissement où le lecteur perçoit, dès lors, un jeu complexe qui reflète celui du rapport entre le mythe/ langage et féminité/écriture. Sa fonction s'éclaire promptement de manière qu'il va octroyer du sens à la forme du texte circulaire et démesuré : «il faut ici revenir à la duplicité de son signifiant, à la fois sens et forme (...) le lecteur vit le mythe à la façon d'une histoire à la fois vraie et irréelle » (Barthes, 1957 : 201-202).

17 En somme, tout consiste à croire que la construction du discours mythique, via l'espace de cette villa, participe activement, en tant que méta-langage, à l'élaboration d'un discours fictionnel au niveau de l'énoncé. Il cumule ainsi, sous "une seconde langue " (Barthes, $1957: 188$ ), tout un imaginaire culturel collectif qui se "réfléchie depuis des millénaires sur les miroirs de la haine» (299). Décidément, la demeure s'impose tel un 
blason qui divulgue l'aspect formel du texte ainsi que son aspect fictionnel : « Le monde entre [alors] dans le langage comme un rapport dialectique d'activités, d'actes humains : il sort $d u$ mythe comme un tableau harmonieux d'essences" (Barthes, 1957: 216-217). Cette réflexion transcendantale semble donc tracer, en dehors du seuil de miroitement, une sorte de jeux formels qui confèrent au récit une configuration expansive et sphérique : "ouverte à toutes les spéculations, épicentre du labyrinthe et de l'énigme» (109). Elle représente, dès lors, une réflexion conjointe du code et de l'énoncé qui se complètent et finissent inévitablement par se rattraper :

le plus merveilleux était que ces univers incomparables, inconciliables en apparence, se rejoignaient et tendaient vers une fin, qui, il [le narrateur] en était certain à présent, compterait parmi les plus grandes joies du lecteur (128-129).

\section{L'exemple de l'aigle harpie}

18 L'aigle empaillé où étaient cachés les documents retrouvés par Ali Khan, qui était luimême dissimulé dans l'une des chambres de la villa, constitue un fragment d'espace restreint accueillant l'histoire quasiment intégrale du récit. Porteur des documents, détenteur et jalon de l'histoire du roman «l'animal empaillé distillait beaucoup de mystère et de trouble» (119). C'est un oiseau sacré qui veille de très près sur la maison majestueuse de Bergagna comme un "témoin hiératique des convenances coloniales, spectateur du ballet ridicule de ces notables» (117). Symbole de la mémoire, il donne cependant l'impression d'être le réservoir d'une œuvre cloîtrée sur elle-même sous forme de jeux clos; un processus d'enchantement ludique qui renvoie aussitôt à un miroir de la même œuvre par mimétisme. Partant, le roman renferme l'histoire du journal qui se trouve dans l'aigle harpie, caché dans la villa, qui contient les documents qui renferment l'histoire du roman etc., et ce de façon circulaire, à l'infini ${ }^{15}$. Ce procédé scriptural consiste à trouver dans un récit » un fragment qui entretient avec l'œuvre qui l'inclut un rapport de similitude [par mimétisme] et qui enchâsse lui-même un fragment qui...et ainsi de suite » (Dällenbach, 1977 : 51). Par ailleurs, nous devons signaler que l'histoire demeure inachevée, puisqu'une partie des carnets de Bergagna a été usurpée par Ali Khan, laissant l'identité de H. Kaïm irrésolue ignorant "jusqu'à sa mort son identité véritable» (304). Le schéma suivant, inspiré de l'ouvrage critique de Dällenbach (1977: 143), illustre d'une manière judicieuse notre représentation de cette réflexion à l'infinie où nous retrouvons, par comparaison, dans le fonctionnement de cette mise en abyme un rapprochement avec le même principe que les poupées gigognes ou les matriochkas.

19 Sachant que dans $\mathrm{E}$, c'est à dire la cellule centrale du schéma, se découvre le récit qui constitue la quasi-totalité du roman où plusieurs histoires se profilent, se renvoyant les unes aux autres, sous forme de jeux circulaires et incessants. L'auteur semble donc s'acharner sur ce "Détournement de texte à transformation minimale " (Genette, 1982: 40), afin de garantir le travail de l'écrivain, qui chercherait à cerner la complexité d'un monde fugitif et insaisissable, en tant que quête illimitée de la création littéraire.

Afin de synthétiser, rappelons, que les réflexions élémentaires peuvent se subdiviser en trois types ou figures essentielles: la réduplication ou dédoublement simple, la réduplication à l'infini et la réduplication spécieuse ou aporistique. La constitution de ses types de mise en abyme s'appuie donc sur les degrés d'analogie

existant entre la mise en abyme de l'énoncé et l'objet qu'elle réfléchit (...) c'est selon qu'elle reflète une même œuvre (par similitude), la même œuvre (par mimétisme) 
ou l'œuvre elle-même (par identité), que la réflexion basale engendre respectivement les types I, II, III (Dällenbach, 1977 : 142).

21 Signalons que, ces trois catégories ont pour fonction de déterminer les mécanismes d'une écriture autocentrique et d'attirer l'attention sur son caractère fictif et son aspect formel. En guise d'illustration, résumons donc dans le tableau suivant de Dällenbach (1977 : 142) les types de mise en abyme analysées jusqu'ici, au niveau du code, dans notre corpus.

\begin{tabular}{|l|l|l|l|}
\hline $\begin{array}{l}\text { Type } \\
\text { Degrés d'analogie }\end{array}$ & $\begin{array}{l}\text { I/ Réduplication } \\
\text { simple }\end{array}$ & $\begin{array}{l}\text { II/ Réduplication à } \\
\text { l'infinie ou répétée }\end{array}$ & $\begin{array}{l}\text { III/ Réduplication } \\
\text { aporistique ou } \\
\text { spécieuse }\end{array}$ \\
\hline $\begin{array}{l}\text { Le journal de Hamid } \\
\text { Kaïm : Par similitude }\end{array}$ & + & - & - \\
\hline $\begin{array}{l}\text { L'aigle harpie : Par } \\
\text { mimétisme }\end{array}$ & - & + & - \\
\hline $\begin{array}{l}\text { Villa de Bergagna LA } \\
\text { KAHENA : Par identité }\end{array}$ & - & - & + \\
\hline
\end{tabular}

Pourtant, tout en exhibant un effet de sérieux délibéré et excessif l'auteur installe, à dessein ludique, une circularité imprécise et imparfaite dans son texte ; c'est ainsi que s'étaye et se divulgue, de manière concise, l'élément important dans le roman qui est l'autocritique ou l'autocontestation du texte. Ce procédé s'obstine à parodier la circularité de l'écriture par amplification du procédé qui s'installe à travers un radotage simulé et voulu, dans une contestation consciente, afin d'attirer l'attention du lecteur "lassé (s) par les mots qui tourbillonnaient maintenant comme des mouches, sans direction définit » (180). En effet, le recours au discours parodique et ludique engage une circularité en colimaçon qui, tout en marquant sa clôture, laisse une brèche au langage pour signifier autre chose que lui-même :

\footnotetext{
- Tu te répètes. (...)

- Je suis libre, dit-il (...) Libre de ne parler de rien, de me répéter, de ressasser, d'aller et de venir dans ce récit, qui est le mien, comprends-tu, le mien!

- Enfermé dans une chambre!

- Je ne commande pas aux ténèbres. Qu'y puis-je, si le monde est tel qu'il est préférable de se cloîtrer chez soi ?

- Rien.

- S'il me plait de revenir sur les événements, d'emberlificoter l'affaire, je le ferais jusqu'à plus soif. Personne n'y peut rien. (270).
}

Se laisse alors percevoir l'enjeu essentiel de cette écriture, qui reposerait sur une double résolution: elle affiche, d'une part, un retour circulaire du texte sur soi, s'affirmant amplement en tant qu'acte créatif. Elle ordonne, d'autre part, des dimensions ludiques, mythique et parodique, qui contaminent et déstabilisent les frontières circulaires du récit autoréflexif. Elle semble, ainsi souscrire une volonté du roman à transcender l'aventure de l'écriture, afin d'intercepter les tourments de l'être humain, en tant que reflet imaginaire d'un monde extérieur au texte. Au demeurant, le lecteur, activement engagé dans le récit, peut bien disposer à volonté sur le sens ou sur la forme comme une "une force réelle, agissante" (127). Il peut intercepter tantôt la 
présence de La Kahéna comme une catharsis, sous forme de "méta-langage» (Barthes, 1957 : 218), pour une ultime consécration de la parole apte à motiver les ferveurs collectives. Tantôt, au contraire, l'effacement du mythe et la vigueur du sens en tant que symbole d'une déesse rebelle : «le résultat de cette alternance sera constant (...) à la fois irréel et plein (...) De même dans le signifiant mythique : la forme y est vide mais présente, le sens y est absent et pourtant plein. » (Barthes, $1957: 196)$.

En affirmant ainsi une singularité scripturale manifeste, l'auteur semble, formellement, vouloir se démarquer de la typologie narcissique adoptée par ses prédécesseurs: " miroirs sans voix, miroirs aux reflets éteints, miroirs sans images, miroirs qui avaient perdu le pouvoir de renvoyer dos à dos les infinis » (111). Il garantit, par conséquent, l'authenticité de son texte en arborant un double discours: sur le langage et sur son temps fragilisant, de la sorte, la tautologie austère d'une écriture éprise de son image.

Suite à cette lecture ambivalente qui a essayé de consolider un caractère paradoxal de la réflexivité, en y décèle une écriture qui soutient à la fois la référence de l'œuvre à soi et la rupture de la circularité excessive du texte sur soi. Nous pouvons avancer, dès lors, que les stratégies réflexives dans LA KAHENA de S. Bachi s'emploient résolument à divulguer, au niveau du code, le caractère fictif de l'écriture en introduisant un retour excessif du texte sur lui-même. Les similitudes que nous avons pu relever, par le biais de la mise en abyme, produisent des degrés d'analogie à des niveaux différents en affirmant, de ce fait, que le récit est en train de se créer et de prendre forme. Cependant, la dimension parodique et ludique, amplement mise en valeur au niveau métatextuel, suggère nettement, par le biais d'une autocritique avérée, d'aller au-delà de cet auto-centrisme égocentrique de l'écriture. Or, l'analyse a pu révéler, en parallèle, que la représentation mythique du personnage féminin de La Kahéna, qui «suggère et [mime] une économie universelle» (Barthes, $1957: 229)$, permet à l'œuvre de transcender ses jeux de formes creux afin de considérer le monde d'où elle découle. Cette constance mythique neutralise donc l'insensé, dans un questionnement incessant, afin de styliser l'essence d'une réalité fuyante et d'intercepter un monde évanescent, en assurant ainsi une conscience et une signifiance au récit. Somme toute, les enjeux de l'écriture réflexive pourraient donc se rattacher, dans ce roman, à leur caractère à la fois transitif et intransitif, accordant une articulation intelligible entre le fond et la forme, en rendant compte d'un dynamisme scriptural singulier, où la littérature ne remet nullement en doute son statut d'art à part entière.

\section{BIBLIOGRAPHIE}

BACHI, Salim, La Kahéna, Gallimard, Paris, 2003.

BACHI, Salim, Autoportrait avec Grenade, Du Rocher, Paris, 2005.

BARTHES, Roland, Mythologies, Le Seuil, Paris, 1957.

BARTHES, Roland, Leçon, "leçon inaugurale de la chaire de sémiologie littéraire du Collège de France“ (prononcée le 7 janvier 1977), Le Seuil, Paris, 1978. 
BUTOR, Michel, "Le roman comme recherche“, dans, Essais sur le roman, Gallimard, Paris, 1960.

DÄLLENBACH, Lucien, Le Récit Spéculaire, Essai sur la mise en abyme, Collection Poétique, Le Seuil, Paris, 1977.

DELEUZE, Gilles, et GUATTARI, Félix, Mille Plateaux, Capitalisme et Schizophrénie 2, Les Éditions de Minuit, 1980.

ELIADE, Mircea, Aspects du mythe, Gallimard, Paris, 1975.

GENETTE, Gérard, Palimpsestes, La littérature au second degré, Le Seuil, Paris, 1982.

GONTARD, Marc, Ecrire La Crise, L'esthétique postmoderne, Collection “Interférences“, Presse universitaire de Rennes, 2013.

LOEHR, Joël, Les grandes notions littéraires, collection U21, Dijon, Edition universitaire, 2010.

SABRI, Nouredine, La Kahéna, un mythe à l'image du Maghreb, L'Harmattan, Paris, 2011.

SRAMEK, Jirí, Pour une Définition du Métarécit, (Études Romanes De BRNO XX), 1990. Pdf, disponible sur, https://www.phil.muni.cz/plonedata/wurj/erb/volumes-11-20/sramek90.pdf, consulté le 14-8- 2017.

\section{NOTES}

1. Nous proposons une graphie en minuscule quand il s'agira de nommer la villa de Bergagna et la reine berbère nommées conjointement "La Kahéna», et nous adopterons une graphie en majuscule et en italique quand il s'agira du titre du roman lui-même » LA KAHENA ».

2. Ou « en abîme ", « $\hat{1}$ » ou «y ", sans changement de sens. Dällenbach propose l'orthographe « abyme» $(1977: 9)$.

3. Cet écrivain (1971) est considéré dans le milieu critique parmi les élites de la nouvelle génération d'écrivains algériens contemporains, que Charles Bonn nomme les Monstres sacrés. Il compte en son actif plusieurs Prix littéraires, entre autres : le Prix Goncourt du premier roman, le Prix Tropiques, la bourse de la découverte Prince Pierre de Monaco et le Prix de la Vocation; ce qui justifie l'influence de ce romancier et sa notoriété dans le milieu littéraire.

4. Nous avons opté personnellement pour la création de ce néologisme, qui nous rappelle celui de Ricardoulien, afin d'octroyer une dérivation au nom propre de $\mathrm{S}$. Bachi.

5. De manière générale, en littérature, la notion de code désigne les règles et les normes qui régissent l'organisation du texte et rend «intelligible le mode de fonctionnement du récit » (Dällenbach, 1977 : 127).

6. Dans Mythologies, Barthes désigne le mythe comme « un système de communication, c'est un message (...) un mode de signification, c'est une forme. Il faudra plus tard poser à cette forme des limites historiques, des conditions d'emploi, réinvestir en elle la société : cela n'empêche pas qu'il faut d'abord la décrire comme forme (...) Ceci ne veut pas dire qu'on doive traiter la parole mythique comme la langue : à vrai dire, le mythe relève d'une science générale extensive à la linguistique, et qui est la sémiologie» $(1957: 181)$.

7. «Reine de la tribu des Djéraoua, La Kahéna a conduit la résistance berbère, vers la fin du VII siècle de l'ère chrétienne, lors des dernières tentatives arabes pour conquérir le Maghreb (...) c'est ainsi qu'apparait cette figure féminine, éclairant presque exclusivement ce que l'on a appelé "les siècles obscurs du Maghreb“ (...) Ce mythe est en fait recréé à partir de l'histoire à laquelle cette figure a échappé par sa complexité (...) Mais au cours du chemin qui a mené La Kahéna de l'Histoire vers le mythe, la littérature va l'intercepter. La figure aura, à partir de la seconde moitié du XIXe siècle (...) une double carrière, l'une historique l'autre littéraire » (Sabri, 2011 : 9). 
8. Le mythe est un méta-langage qui se désigne comme parole, comme langage à «double fonction : il désigne et il notifie, il fait comprendre et il impose » (Barthes, 1957 :190).

9. C'est un aspect de l'écriture «qui consiste à définir le même par le même (...) un évanouissement à point venu, une aphasie salutaire (...) [qui] fonde un monde mort, un monde immobile ». (Barthes, 1957 : 227).

10. Nous proposons de désigner les références relatives au roman de Salim Bachi, LA KAHENA, uniquement par leurs pages correspondantes, afin d'éviter la redondance du nom de l'auteur.

11. «Les renvois au texte (code) sont en fait un phénomène courant, et ils caractérisent les genres littéraires les plus divers. Les récits littéraires intègrent normalement des énoncés personnels rapportés, soit à l'auteur (le domaine du métadiscours), soit aux agents de la narration (le domaine du métarécit)». (Jirí Sramek, 1990 : 6).

12. La parodie vient du grec qui « signifie précisément " chant à côté du chant", chant en marge du chant (...) on peut définir un texte parodique comme réécriture, transformation ludique d'un autre texte, d'un système littéraire reconnaissable. Et notamment donc comme la réécriture d'un certain nombre de codes, de conventions, de normes génériques (...) dévoyés, détournés de leurs fins premières, parce qu'ils sont imités dans une visée comique, polémique ou satirique » (Loehr, 2010 : 115).

13. La notion d'écriture postmoderne est présentée en général comme « réécriture de la modernité ». Elle se propose comme un travail anamnestique, vu la manière dont elle revisite la mémoire et le passé littéraire. L'écriture postmoderne s'avère être donc, selon les critiques, très opérationnelle, sans être décisive et unique, dans la mise en œuvre de la littérature contemporaine.

14. Selon Mircea Eliade, le mythe « raconte une histoire sacrée; relate un événement qui a eu lieu dans le temps primordial, le temps fabuleux des "commencements"» (1988:15).

15. On retrouve cette forme de mise en abyme, essentiellement, en peinture. Cependant, Les Faux Monnayeurs de Gide (1925), qui a été soutenu par Dällenbach dans son ouvrage (1977), représente un exemple concret de par l'enchâssement infini de l'œuvre dans l'œuvre.

\section{RÉSUMÉS}

L'examen des stratégies réflexives de la mise en abyme dans LA KAHENA ${ }^{1}$ de Salim Bachi, roman éponyme d'une reine guerrière berbéro-maghrébine, divulgue différentes techniques de construction et de fonctionnement textuels. Ces multiples dispositions et stratégies scripturales finissent, souvent, par se dévoiler comme une allégorisation de l'écriture, visiblement autoréflexive. Néanmoins, solidaire avec une intonation parodique, la présence de la dimension mythique, au niveau métatextuelle, s'ordonne comme une prise de conscience du texte et une catharsis qui permet au langage de s'accommoder « sur le signifiant du mythe comme sur un tout inextricable de sens et de forme " (Barthes, 1957 : 201). Le mythe se dresse, de ce fait, tel un miroir qui instruit sur le passé en harmonisant le présent. Ainsi, le roman de Bachi qui semble se démarquer des apories du structuralisme, sans pour autant s'en écarter totalement, arbore manifestement une manière singulière et complexe de réfléchir sur le langage et l'esprit d'un monde possible, dans un rapport sensible avec l'expérience humaine.

The review of the reflexive strategies of mise-en-abîme in LA KAHENA by Salim Bachi, the eponymous novel of a Berber-Maghrebian warrior queen, reveals different techniques of textual 
construction and functioning. These several dispositions and scriptural strategies often end up as a suggestive metaphor and allegorization for writing, visibly self-reflexive. Nevertheless, in solidarity with a parodic and playful intonation, the presence of the mythical dimension, at the metatextual level, is ordered as an awareness of the text and a catharsis that allows the language to accommodate "on the signifier of the myth as on an inextricable whole of sense and form "(Barthes, 1957: 201). Thus, Bachi's novel which seems to differ from the aporias of structuralism, without departing totally from it, clearly displays a singular and complex way of reflecting on the language and spirit of a possible world, in a sensitive relationship with human experience.

INDEX

Mots-clés : réflexivité, mise en abyme, mythe, code, métatextualité

الانعكاسية, التناص, أسطورة, الشفرة, ميتانصية فهرس الكلمات المفتاحية:

Keywords : reflexivity, mise-en-abîme, myth, code, metatextuality

\section{AUTEURS}

\section{LEILA OULEBSIR}

Doctorante, Littérature française, Université Alger 2

\section{SABRINA FATMI}

Université Alger 2 